\title{
Überprüfung des Impfschutzes beim gesunden Erwachsenen
}

Wolfensberger, A ; Hatz, Christoph ; Funk, M ; Rampini Speck, S

DOI: https://doi.org/10.1024/1661-8157/a000981

Other titles: Verification of vaccine protection in healthy adults

Posted at the Zurich Open Repository and Archive, University of Zurich

ZORA URL: https://doi.org/10.5167/uzh-67486

Journal Article

Accepted Version

Originally published at:

Wolfensberger, A; Hatz, Christoph; Funk, M; Rampini Speck, S (2012). Überprüfung des Impfschutzes beim gesunden Erwachsenen. Praxis, 101(14):887-898.

DOI: https://doi.org/10.1024/1661-8157/a000981 
DOI 10.1024/1661-8157/a000981

((PX_14_Rubrik CME))

\section{Überprüfung des Impfschutzes beim gesunden Erwachsenen}

Die Überprüfung des Impfschutzes ist Teil der «Check-Up»-Untersuchung in der hausärztlichen Praxis. Einerseits sollte auch bei Erwachsenen eine nicht durchgeführte, empfohlene Basisimmunisierung nachgeholt oder unvollständige Impfungen vervollständigt werden, anderseits bedürfen gewisse Impfungen einer Auffrischung (Booster).

Ziel jeder Impfung ist der Schutz des Individuums und des Kollektivs vor potenziell gefährlichen Infektionskrankheiten. Das Kollektiv wird durch die sogenannte Herdenimmunität geschützt. Diese bezeichnet den Effekt, dass durch Impfung (oder auch durch Krankheit) erzeugte Immunität gegen einen bestimmten Erreger innerhalb einer Population auch nicht immune Individuen schützt, da sich der Erreger nicht mehr ausbreiten kann. Noch immer ist die Durchimpfungsrate vor allem bei Erwachsenen in der Schweiz nicht optimal. Bei den Masern beispielsweise liegt die Durchimpfungsrate der Gesamtbevölkerung bei 82\%, für eine Ausrottung der Masern, bzw. für Erreichen der Herdenimmunität, müsste die Durchimpfungsrate $>95 \%$ liegen. Durch eine regelmässige Überprüfung des Impfschutzes anhand der Impfdokumente durch Fachpersonen im Gesundheitswesen kann die Durchimpfungsrate verbessert werden.

In diesem CME werden die beim Erwachsenen empfohlenen Impfungen besprochen; auf die Impfungen bei Kindern und auf Reiseimpfungen wird nicht eingegangen.

Die Empfehlungen in diesem CME richten sich nach dem schweizerischen Impfplan [1]. Dieser wird jährlich durch die Eidgenössische Kommission für Impffragen (EKIF) in Zusammenarbeit mit dem Bundesamt für Gesundheit (BAG) und der Swissmedic aktualisiert. 


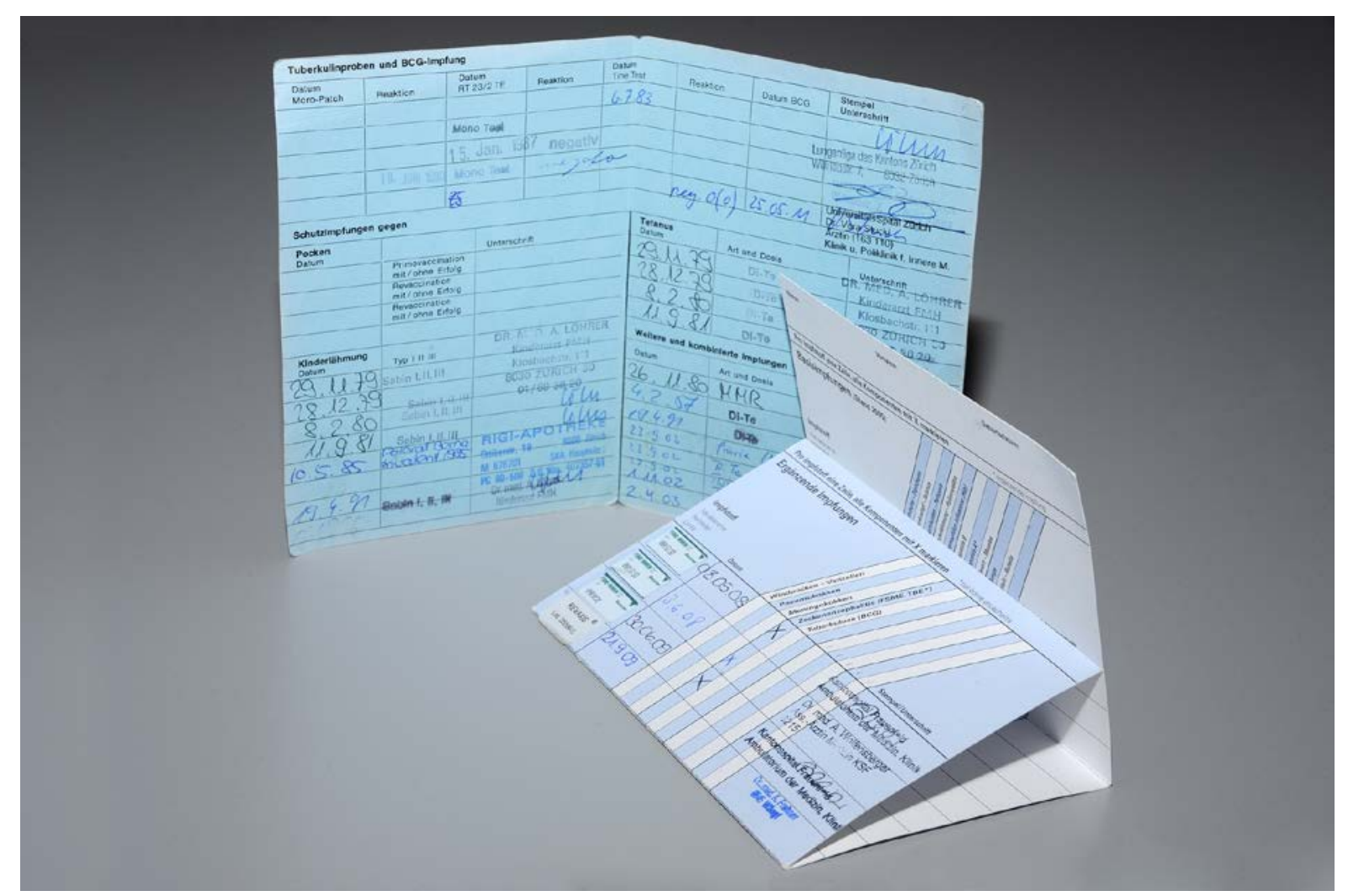

((Bild für 1. Seite))

Legende : Zwei verschiedene Generationen von Impfdokumenten

Im Artikel verwendete Abkürzungen:

BAG Bundesamt für Gesundheit

EKIF Eidgenössische Kommission für Impffragen

FSME Frühsommermeningoenzephalitis

HPV Humane Papillomaviren

MMR Masern/Mumps/Röteln

RQW Riss-Quetsch-Wunde

VZV Varizella zoster Virus 
Damit der Patient individuell beraten werden kann, bedarf es folgender Grundkenntnisse.

\section{Generelle Impfempfehlungen für Erwachsene ((Ü2))}

Im Schweizerischen Impfplan wird unterschieden zwischen empfohlenen Basisimpfungen, empfohlenen ergänzenden Impfungen (für Erwachsene nur HPV) und empfohlenen Impfungen für Risikogruppen (Tab. 1). Diese Impfungen werden von der Krankenkasse übernommen.

\begin{tabular}{|c|c|}
\hline Empfohlene Basisimpfungen für alle Altersgruppen & $\begin{array}{ll}\text { - } & \text { Diphtherie/Tetanus/Pertussis } \\
\text { - } & \text { Masern/Mumps/Röteln (MMR) } \\
\text { - } & \text { Polio }\end{array}$ \\
\hline $\begin{array}{l}\text { Empfohlene Basisimpfungen und ergänzende } \\
\text { Impfungen für bestimmte Altersgruppen }\end{array}$ & 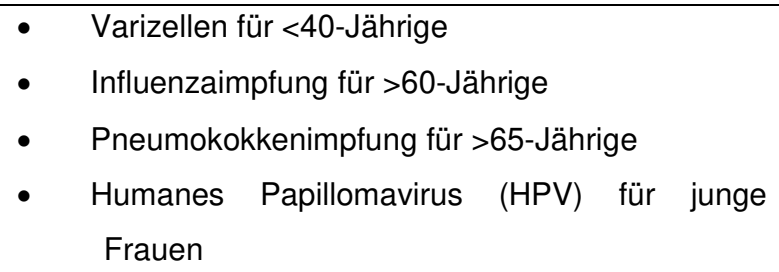 \\
\hline Für Risikogruppen empfohlene Impfungen & $\begin{array}{ll}\text { - } & \text { Hepatitis A und B } \\
\text { - } & \text { FSME } \\
\text { - } & \text { Grippe } \\
\text { - } & \text { Meningokokken } \\
\text { - } & \text { Pneumokokken } \\
\text { - } & \text { Varizellen } \\
\text { - } & \text { Tollwut } \\
\text { - } & \text { Andere Reiseimpfungen wie Gelbfieber, Typhus, } \\
\text { - } & \text { Impanische Enzephalitis, Cholera } \\
& \text { Gesunghen für Beschäftigte im } \\
\end{array}$ \\
\hline
\end{tabular}

Tabelle 1

Immunisierungsarten ((Ü2))

Aktive Immunisierung: Bei der aktiven Impfung wird durch die Injektion oder Ingestion von Tot- oder Lebendimpfstoffen (und somit von Antigenen) das Immunsystem zur Bildung von spezifischen Antikörpern angeregt. Eine spätere Exposition durch den Erreger führt via Aktivierung von Gedächtniszellen zu einer schnellen Immunantwort und zur Abwehr der Erreger.

Passive Immunisierung: Hier werden dem Körper passiv spezifische Antikörper zugeführt, die sofort, dafür nur kurzzeitig (Wochen bis Monate) wirken.

Grundimmunisierung: Aufbauen eines erstmalig genügenden Impfschutzes und eines immunologischen Gedächtnisses durch eine bestimmte Anzahl Impfungen in einem vorgegebenen Mindestabstand. Die Grundimmunisierung sollte bei den meisten Impfungen im Kindesalter erfolgen.

Nachholimpfung: Vervollständigung oder Durchführung der Grundimmunisierung von Erwachsenen, die als Kind ungenügend oder gar nicht geimpft wurden.

Auffrischimpfung (Booster): Gabe eines (teilweise geringer dosierten) Impfstoffes zum erneuten Erreichen eines genügenden Antikörperspiegels durch Reaktivierung des immunologischen Gedächtnisses, nachdem eine Grundimmunisierung lege artis durchgeführt wurde. 


\section{Wirkstoffe ((Ü2))}

Es wird unterschieden zwischen Lebend- und Totimpfungen. $\mathrm{Zu}$ den Lebendimpfungen gehören die Masern/Mumps/Röteln-, die Varizellen-, die Gelbfieber-, die Typhus- und die BCG-Impfung. Diese Erreger sind attenuiert, also abgeschwächt, sie können sich noch vermehren, jedoch keine Krankheit mehr auslösen. Grundsätzlich werden Lebendimpfungen subkutan verabreicht.

Totimpfstoffe bestehen aus abgetöteten Erregern (z.B. die bei uns gebräuchliche Polioimpfung), antigenen Untereinheiten (z.B. Hepatitis B) von Organismen oder aus Toxoiden (Diphtherie, Tetanus). Diese Impfungen erfolgen meist intramuskulär, können aber bei z.B. antikoagulierten Patienten zum Teil auch subkutan erfolgen, wobei dann die Immunantwort möglicherweise suboptimal ausfällt und mit vermehrten lokalen Nebenwirkungen zu rechnen ist.

Tab.2: Impfstoffarten

\begin{tabular}{|l|l|}
\hline Lebendimpfstoffe & - MMR \\
& - Varizellen \\
& - Typhus \\
& - Gelbfieber \\
& - BCG \\
\hline Totimpfstoff aus inaktivierten Erregern & - Polio \\
& - FSME \\
& - Hepatitis A \\
& - Influenza \\
& - Pneumokokken \\
& - Meningokokken \\
& - Jollwut \\
\hline Totimpfstoff aus Toxoiden & - Diphtherie \\
\hline Totimpfstoff aus rekombinanter DNA & - Tetanus \\
\hline
\end{tabular}

\section{Jede Impfung zählt! ((Ü2))}

Grundsätzlich gilt bei Impfungen: Jede Impfdosis zählt, es muss nie von vorne begonnen werden! Bei den meisten Impfungen (MMR, Hepatitis etc.) kann trotz jahrelanger Latenz die Impfserie einfach fortgesetzt werden. Bei einigen Impfungen ( $\mathrm{dTp}_{\mathrm{a}}$, Polio) ist das Impfschema komplexer und Entscheidungshilfen bzgl. Nachholimpfungen müssen dem schweizerischen Impfplan entnommen werden. Vereinfacht gesagt brauchen ungeimpfte Erwachsene drei Impfungen und unvollständig geimpfte Erwachsene ein bis drei Impfungen, sodass insgesamt drei Impfungen nach dem ersten Lebensjahr verabreicht werden.

\section{Gleichzeitige Verabreichung von Impfungen ((Ü2))}

Die gleichzeitige Verabreichung verschiedener Impfungen (auch von Lebendimpfstoffen) ist möglich, die Impfstoffe sollten jedoch an verschiedenen Stellen appliziert werden. Sind Intervalle zwischen den Impfungen gewünscht, wird bei Lebendimpfstoffen mindestens vier Wochen empfohlen. 


\section{Vorgehen bei der Überprüfung des Impfschutzes ((Ü1))}

In Kenntnis der vorgängig diskutierten Grundlagen basiert die individuelle Impfempfehlung einerseits auf der Prüfung des Impfausweises zum Nachweis durchgeführter Impfungen, anderseits auf anamnestischen Angaben bezüglich Herkunft und durchgemachter Krankheiten. Kontraindikationen müssen abgeklärt werden. In Spezialfällen kann eine gezielte Antikörperbestimmung durchgeführt werden.

\section{Prüfung des Impfausweis und Impfanamnese ((Ü2))}

Das wichtigste Dokument zur Überprüfung des Impfschutzes ist der Impfausweis, ergänzt durch Kontrollen des militärischen Dienstbüchleins, falls vorhanden. Gelegentlich stammen die Dokumente aus anderen Ländern und sind in anderen Sprachen abgefasst. Trotzdem sind sie aufgrund der internationalen Nomenklatur oft zu entziffern und liefern wichtige Daten. Erscheint der Patient ohne seinen Impfausweis in der Praxis, sollte insistiert werden, dass er diesen bei der nächsten Konsultation mitbringt. Dies nicht nur, um frühere Impfungen einzusehen, sondern auch, um aktuell durchgeführte Impfungen zu dokumentieren.

Eine besondere Herausforderung stellen Patienten dar, die keine oder eine unvollständige Dokumentation ihres Impfstatus vorweisen können. Generell gilt, dass man von einer erfolgten Grundimmunisierung in der Kindheit ausgehen kann, sofern die Patienten aus einem europäischen Land stammen und nicht einer Randgruppe angehören. Ausnahmen bilden Kinder von Impfgegnern, wobei die Betroffenen meist über die nicht erfolgte Grundimmunisierung Bescheid wissen. Die WHO bietet genaue Übersichten über die Impfprogramme und die Durchimpfungsrate der verschiedenen Impfungen in allen Ländern der Welt [2]. Anamnestische Angaben zu früheren Impfungen oder durchgemachten Kinderkrankheiten (v.a. Röteln und Masern) sind meist nicht zuverlässig, ausser bei Varizellen, wo häufig auch noch Narben sichtbar sind.

Immer wieder trifft man aber auch auf Patienten, die aufgrund häufiger Wohnungs- oder Arztwechsel auch bei den Basisimpfungen nur ungenügend grundimmunisiert worden sind oder die längere Zeit keine Auffrischimpfung erhalten haben. Das heisst, jeder Impfausweis muss genau studiert werden! Dies kann kaum zwischen «Tür und Angel» passieren, der Arbeitsaufwand beträgt rasch fünf bis zehn Minuten.

\section{Spezifische Anamnese und Evaluation Risikofaktoren ((Ü2))}

Anhand von Alter, Grunderkrankungen und speziellen Risikofaktoren wird die Indikation für empfohlene Impfungen für bestimmte Alters- und Risikogruppen geprüft. Die empfohlene Basisimpfungen und ergänzende Impfungen für bestimmte Altersgruppen sind in Tabelle 1 aufgeführt.

Folgende anamnestische Hinweise müssen berücksichtigt werden:

- Bestehen Risiken im Freizeitverhalten oder in Zusammenhang mit dem Wohnort, wie z.B eine Zeckenexposition, dann sollte der Patient gegen FSME geimpft werden.

- $\quad$ Sind Reisen geplant, muss der Patient über länderspezifische Risiken aufgeklärt und entsprechend geimpft werden.

- $\quad$ Bestehen sexuelle Risikofaktoren (Homosexualität, Geschlechtsverkehr mit Hepatitis-B-Patient) soll eine Hepatitis-B-Impfung erfolgen.

- Beschäftigte im Gesundheitswesen, im Sozialdienst oder bei der Polizei sollen gegen Hepatitis B, Kanalisationsarbeiter gegen Hepatitis A geimpft werden.

- Tierärzte, Tierpfleger, Personen im Umgang mit Fledermäusen sollen in der Regel eine Tollwutimpfung erhalten.

- $\quad$ Bestehen medizinische Grunderkrankungen, muss die Indikation für eine Influenza-, Pneumokokken- oder Meningokokken-Impfung geprüft werden. 


\section{Antikörperbestimmung ((Ü2))}

Es gibt nur wenige Indikationen für eine Bestimmung der spezifischen Antikörper vor oder nach einer Impfung:

Varizella-zoster-Virus (VZV): Bei negativer Krankheitsanamnese sollten die Varizellen-Antikörper vor einer geplanten VZV-Impfung bestimmt werden. Die Durchseuchungsrate ist so hoch, dass die meisten Impfkandidaten auch bei blander Anamnese VZV positiv sind.

Masern, Mumps, Röteln: Masern-, Mumps- oder Röteln-Serologien werden generell nur bei Schwangeren ohne dokumentierte Impfung empfohlen (diese dienen als Referenzwerte, sollte später der Verdacht auf eine Erkrankung auftauchen), bei allen anderen wird die trivalente Impfung empfohlen, auch wenn anamnestisch eine Erkrankung durchgemacht wurde (siehe unten). Die Empfehlung beruht darauf, dass einerseits keine Gefahr einer Hyperimmunisierung besteht, anderseits die Bestimmung von Serologien aufwendig und kostenintensiv ist, Verzögerungen nach sich zieht und auch nicht sehr sensitiv ist.

Tetanus: Selten sollten die Tetanus-Antitoxin-Titer bestimmt werden, und zwar dann, wenn ein unbekannter Impfstatus vorliegt und eine Hyperimmunisierung vermieden werden soll (siehe unten). Bei ausgeprägter Unverträglichkeit von früheren Impfungen können ausnahmsweise auch Antikörper bestimmt werden, um einen genügenden Impfschutz zu dokumentieren.

Serologische Erfolgskontrollen nach einer Impfung sind nur bei Beschäftigten im Gesundheitswesen indiziert (nach Hepatitis B- und nach Varizellen-Impfung) oder als Erfolgskontrolle bei Verdacht auf mangelnde Wirkung, z.B. bei Immunsupprimierten (nach Hepatitis A und B-, MMR-, Pneumokokken-, Varizellen-Impfung). Grundsätzlich sollten sie vier bis sechs Wochen nach Beendigung der Grundimmunisierung oder Booster erfolgen. Erschwerend kommt hinzu, dass bei vielen Impfungen der schützende Antikörpertiter nicht bekannt ist.

\section{Kontraindikationen für Impfungen ((Ü2))}

Absolute Kontraindikation für eine Impfung ist eine stattgehabte anaphylaktische Reaktion auf eine frühere Impfung oder schwere neurologische Komplikationen (wie z.B. ungeklärte Enzephalopathie innerhalb einer Woche) nach einer Impfung. Lebendimpfstoffe sind kontraindiziert bei Immuninkompetenten und Schwangeren. Nach einer Lebendimpfung sollte eine Frau während einem Monat nicht schwanger werden. Inaktivierte Impfstoffe können bei Schwangeren verabreicht werden, wenn die Indikation klar gegeben ist. Während akuten, schweren Erkrankungen sollte keine Impfung erfolgen, leichtere Erkrankungen ohne Fieber sind kein Hinderungsgrund.

\section{Nebenwirkungen ((Ü2))}

Lokale Nebenwirkungen wie Schmerz, Rötung und Schwellung sind die häufigsten unerwünschten Impferscheinungen. Sehr viel seltener kommen Granulome und Nekrosen, sowie eine Brachialneuritis oder regionale Lymphadenopathien vor. Häufige systemische Nebenwirkungen sind Fieber und Exantheme (2-5\% v.a. nach MMR). Sehr selten kommt es zu einer Immunthrombopenie (nach Masernimpfung) oder zur Anaphylaxie. Mit Impfungen in Zusammenhang gebracht, aber nach dem heutigen Stand des Wissens nicht kausal mit Impfungen assoziiert, wurde das Auftreten verschiedenster Erkrankungen wie z.B. Fälle von Guillain-BarréSyndrom, Multipler Sklerose, Autismus, Diabetes, plötzlicher Kindstod.

\section{Die Impfaufklärung ((Ü2))}

Impfungen sind juristisch gesehen Eingriffe in die körperliche Integrität. Jeder Patient muss vor der Impfung über deren Nutzen und allfällig zu erwartende Nebenwirkungen aufgeklärt werden. In der Krankengeschichte muss eine kurze Dokumentation über die erfolgte Aufklärung gemacht werden.

Kontraindikationen oder Einschränkungen für Impfungen (Allergien, mögliche Schwangerschaft, Medikamente wie Immunsuppressiva oder Antikoagulanzien, andere Gründe für eine Immunsuppression etc.) sollten auch bei in der Praxis bekannten Patienten nochmals erfragt werden. 


\section{Impfzwang ((Ü2))}

Grundsätzlich besteht auch bei absoluter Indikation, z.B. bei im gleichen Haushalt mit Immunsupprimierten Lebenden, bei Beschäftigten im Gesundheitswesen etc., kein Impfzwang. Das bedeutet, dass niemand, auch bei erhöhtem Risiko für sich selbst oder für andere, zu einer Impfung gezwungen werden kann. Hier ist die umfassende Aufklärung sehr wichtig, eine Dokumentation in der Krankengeschichte ebenfalls.

\section{Impfdokumentation ((Ü2))}

Seit 2008 steht ein neuer eidgenössischer Impfausweis zur Verfügung, in dem Impfungen übersichtlich dokumentiert werden können. Er löst die älteren kantonalen Dokumente ab. Die Dokumentation besteht aus der Angabe von Krankheit und verwendetem Impfstoff. Der dem Impfstoff beigelegte Kleber muss ins Dokument eingeklebt werden (Impfstoff/Lot Nummer) Ein internationaler Impfausweis (erhältlich bei der WHO) wird nur für die Gelbfieberimpfung, die übrigens auch nur durch berechtigte Ärzte verabreicht werden darf, benötigt.

Seit 2011 gibt es die Impfsoftware Viavac [3] zur Verwaltung von Impfungen für Patient und Hausarzt. Patienten können das zugehörige iPhone-App erwerben, ihre Impfungen selbst dokumentieren oder ihre Dokumentation mit der Dokumentation ihres Hausarztes synchronisieren bzw. diese durch den Arzt validieren lassen. Das App und die zugehörige Internetseite (www.meineimpfungen.ch) hat eine Check-Funktion über die Vollständigkeit der Impfungen und eine Erinnerungsfunktion für noch durchzuführende Impfungen.

\section{Bezahlung der Impfung ((Ü2))}

Grundsätzlich werden Impfungen von der Krankenkasse übernommen, wenn die Indikation im Artikel 12a der KLV (Krankenpflege-Leistungsverordnung) aufgeführt ist. Die KLV richtet sich wiederum nach den Empfehlungen des schweizerischen Impfplans. Ausser bei gewissen Zusatzversicherungen gilt jedoch auch bei Impfungen der obligatorische Selbstbehalt, weshalb die Impfungen von ansonsten gesunden Erwachsenen de facto oft selbst bezahlt werden müssen. 


\section{Diphtherie/Tetanus/Pertussis ((Ü3))}

Zur Grundimmunisierung gegen Diphtherie und Tetanus benötigt ein Erwachsener drei Impfdosen, sowie Auffrischimpfungen alle 20 Jahre (neu seit 2012) bis zum Alter von 65 Jahren, und Auffrischimpfungen alle 10 Jahre ab dem Alter von 65 Jahren. Dieses Schema mit verlängertem Intervall der Auffrischimpfungen für jüngere Erwachsene berücksichtigt die Gefahr der Hyperimmunisierung mit starken lokalen und systemischen Nebenwirkungen. Andererseits zollen die Intervalle von zehn Jahren der reduzierten Persistenz von Antikörpern bei älteren Menschen (Immunseneszenz) Rechnung. Bei immunsupprimierten Patienten, Reisenden in Diphtherie-Endemiegebiete oder Entwicklungsländer, in denen es möglicherweise keinen Zugang zur TetanusProphylaxe gibt, ist die Auffrischimpfung weiterhin alle zehn Jahre empfohlen.

Ist ein Erwachsener in der Kindheit nur unvollständig geimpft worden, sind die notwendigen individuellen Empfehlungen für die Nachholimpfungen dem schweizerischen Impfplan 2012 zu entnehmen.

Ist der Impfschutz unbekannt und soll eine Hyperimmunisierung vermiedern werden, ist eine einmalige Impfung mit anschliessender Bestimmung der Antikörper gegen Tetanustoxin empfohlen.

Ebenfalls neu ab 2012 sind die Richtlinien für eine Tetanus-Prophylaxe bei Verletzungen: Eine Tetanus Auffrischimpfung sollte bei kleinen, oberflächlichen Wunden durchgeführt werden, wenn die letzte Impfung mehr als 20 Jahre zurückliegt. Bei allen anderen Wunden soll ein Tetanus-Booster erfolgen, wenn die letzte TetanusImpfung mehr als zehn Jahre zurückliegt.

Für Pertussis wird neu (seit 2012) für Erwachsene im Alter von 25 bis 29 Jahren eine Auffrischimpfung oder eine Primovakzination (in Form einer Einzeldosis $\mathrm{dTp}_{\mathrm{a}}$ ) empfohlen. Eine Auffrischimpfung ebenfalls allen Personen empfohlen, die regelmässigen Kontakt zu Säuglingen unter sechs Monaten haben. Das minimale Intervall zur letzten dT-Impfung sollte zwei Jahre betragen, bei geplanter Schwangerschaft oder bei regelmässigem Kontakt mit Säuglingen kann dieses Intervall jedoch auf vier Wochen verkürzt werden.

Diese Anpassung im schweizerischen Impfplan berücksichtigt, dass noch ungeimpfte Säuglinge oft von ihren Eltern oder Betreuungspersonen mit Pertussis angesteckt werden und darum eine zusätzliche Dosis des Impfstoffes mit konsekutiv verbessertem Impfschutz Sinn macht.

\section{Poliomyelitis ((Ü3))}

Die Grundimmunisierung eines nicht geimpften Erwachsenen umfasst drei Dosen des Totimpfstoffes (intramuskuläre Poliovakzine IPV). Unvollständig geimpfte Erwachsene benötigen ein bis drei Impfungen, sodass insgesamt drei Impfungen nach dem ersten Lebensjahr verabreicht wurden. Poliomyelitis-Auffrischimpfungen für Erwachsene sind nur bei Reisen in Endemiegebiete (siehe BAG-Bulletin/Reisemedizin) nötig und sind dann alle zehn Jahre durchzuführen.

\section{Masern/Mumps/Röteln ((Ü3))}

Bei allen Erwachsenen mit Jahrgang 1964 oder jünger können Nachholimpfungen nötig sein. Die Nachholimpfung umfasst nach Impfanamnese eine bis zwei Dosen im Abstand von mindestens einem Monat. Bei Patienten mit Jahrgang 1963 oder älter geht man von einer Immunität aus. Dies gilt jedoch nicht bei Beschäftigten im Gesundheitswesen, dort sollte bei unvollständiger Impfanamnese der Immunstatus serologisch überprüft oder die Beschäftigten unabhängig von ihrem Jahrgang geimpft werden. Eine anamnestisch durchgemachte Masern- oder Rötelnerkrankung entbindet nicht von der Notwendigkeit einer Impfung, da oft eine andere virale Erkrankung mit 
Exanthem als Masern- oder Rötelninfektion verkannt wird. Nur bei einem anamnestisch durchgemachten Mumps mit dem typischen Beschwerdebild kann man von einer Impfung absehen.

\section{Varizellen ((Ü3))}

Eine Nachholimpfung ist bei allen jungen Erwachsenen ( $<40$ Jahren), die Varizellen nicht durchgemacht haben, empfohlen. Ist die Anamnese negativ, sollten vor der Impfung IgG-Antikörper bestimmt werden. Die Impfung erfordert zwei Dosen im Abstand von mindestens einem Monat. Im Rahmen der ergänzenden Impfung für Risikogruppen ist die Impfung auch für >40-Jährige empfohlen (siehe Listen im schweizerischen Impfplan).

\section{Humanes Papillomavirus (HPV) ((Ü3))}

Jungen Frauen zwischen 11 und 19 Jahren wird empfohlen, die Impfungen durchzuführen. Das Impfschema umfasst drei Dosen für über 15-Jährige. Für unter 15-Jährige genügen zwei Dosen (neu seit 2012).

Für Risikogruppen ergänzende Impfungen: ((Ü2))

\section{FSME ((Ü3))}

Die FSME-Impfung wird allen Erwachsenen, die in Endemiegebieten wohnen oder sich zeitweise dort aufhalten, empfohlen. Die Grundimmunisierung umfasst drei Dosen. Auffrischimpfungen sind alle zehn Jahre notwendig.

\section{Influenza ((Ü3))}

Die saisonale Grippeimpfung ist grundsätzlich bei allen Erwachsenen über 65 Jahre, bei Patienten mit chronischen Erkrankungen, bei Schwangeren ab dem 2. Trimenon und Wöchnerinnen bis vier Wochen post partum und bei allen Personen, die mit Patienten der vorher genannten Kategorien regelmässigen Kontakt haben, empfohlen.

\section{Hepatitis A ((Ü3))}

Die Hepatitis-A-Impfung wird neben Reisenden in Endemiegebiete u.a. auch Drogenabhängigen, Personen mit beruflichem Kontakt zu Drogenabhängigen oder Kleinkindern, Mitarbeitern in Institutionen für Schwerbehinderte, homosexuellen Männern, Kanalisationsarbeitern und Patienten mit chronischen Lebererkrankung empfohlen.

\section{Hepatitis B ((Ü3))}

Bei Kindern und Jugendlichen zählt die Hepatitis-B-Impfung zu den Basisimpfungen - sie ist für alle empfohlen. Bei Erwachsenen gehört sie zu den für bestimmte Risikogruppen empfohlenen Impfungen. Dazu gehören: Medizinal- und Laborpersonal, Sozialberufe im Umgang mit Randständigen, Polizei und Feuerwehr, Dialysepatienten, Drogenabhängige, Personen mit häufig wechselnden Sexualpartnern, homosexuelle Männer, enge Kontaktpersonen von HBs-Ag-Trägern, Reisende in Endemiegebiete etc. Die obligatorische Krankenversicherung übernimmt die Kosten bei allen Indikationen ausser bei Reisenden. Bei NichtRisikopersonen braucht es drei Impfdosen, eine Auffrischimpfung oder eine Laborkontrolle ist nicht indiziert. Eine serologische Impfkontrolle ist nur bei Risikopersonen notwendig, von einem lebenslangen Impfschutz kann ausgegangen werden, wenn einmalig ein Anti-HBs-Titer von >100 IE/I erreicht worden ist.

\section{Meningokokken ((Ü3))}

Die Meningokokkenimpfung ist indiziert bei medizinischen und beruflichen Risikofaktoren, sei dies eine Asplenie, bestimmte Formen der Immundefizienz, Angestellte eines mikrobiologischen Labors etc. (umfassende Listen siehe schweizerischer Impfplan). Da ein möglichst breiter Schutz angestrebt wird, sollte der quadrivalente Konjugatimpfstoff (Menveo®) verwendet werden. Zur Grundimmunisierung werden für Personen mit einem stark 
erhöhten Risiko für invasive Meningokokkenerkrankungen zwei Dosen Menveo® im Abstand von vier bis acht Wochen benötigt. Für Personen mit erhöhtem Expositionsrisiko (z.B. im Personal im Forschungslabor, Mikrobiologie etc.) wird nur eine Impfung benötigt. Rekruten werden mit einem monovalenten Impfstoff gegen Meningokokken der Serogruppe C geimpft. Auffrischimpfungen der quadrivalenten Impfung sollen alle fünf Jahre mit dem quadrivalenten Polysaccharidimpfstoff (Mencevac®) durchgeführt werden, sofern das Expositionsrisiko bzw. das Risiko für eine invasive Erkrankung fortbesteht.

\section{Pneumokokken ((Ü3))}

Auch hier besteht eine breite Indikationspalette, die den Listen des schweizerischen Impfplans zu entnehmen ist. Grundsätzlich ist die Impfung für Personen jeden Alters mit erhöhtem Risiko einer Pneumokokkenerkrankung sowie für Personen über 65 Jahre indiziert. Die Grundimmunisierung erfolgt mit einer Dosis, eine Auffrischimpfung ist bei Personen indiziert, die während mehr als fünf Jahren keine Impfung erhalten haben und die bei der ersten Dosis jünger als 65 Jahre alt waren. Die Impfung erfolgt mit einem Polysaccharidimpfstoff (z.B. Pneumovax®), der Konjugatimpfstoff (Prevenar ${ }^{\circledR} 13$, Nachfolge-Impfstoff von Prevenar® 7) ist aktuell in der Schweiz nur zur Impfung von Kleinkindern zugelassen.

\section{Tollwut ((Ü3))}

Die Tollwutimpfung ist nur bei Tierärzten, Tierpflegern, Personal in Diagnostiklabors und Personen mit Kontakten zu Fledermäusen indiziert. Ausserdem wird sie als Reiseimpfung in bestimmten Risikosituationen empfohlen.

\section{BGC-Impfung ((Ü3))}

Es gibt keine Indikation für eine BCG-Impfung bei Personen älter als 1 Jahr!

\section{Impfungen vor Auslandreisen ((Ü3))}

Für Auslandreisende bestehen Empfehlungen des Expertenkomitees für Reisemedizin, die auf entsprechenden Internetseiten wie der Internetseite des BAG (http://www.bag.admin.ch/themen $\rightarrow$ Krankheiten und Medizin $\rightarrow$ Infektionskrankheiten $\rightarrow$ Impfungen $\rightarrow$ Reisemedizin), Tropimed® (www.tropimed.com für Fachpersonen, kostenpflichtig), oder www.safetravel.ch (für Laien) verfügbar sind. Grundsätzlich gibt es Impfungen gegen Gelbfieber, Typhus, Tollwut, japanische Enzephalitis und Cholera. Allenfalls sind aufgrund der Risikosituation im bereisten Land auch bisher nicht durchgeführte Impfungen aus der Rubrik «Impfungen für Risikogruppen» (wie z.B. Hepatitis A, FSME, Polio, Meningokokken) nötig. 
Das Angebot an Impfstoffen ändert sich ständig. In der folgenden Tabelle wurden einige Impfstoffe als Beispiele aufgelistet, diese Auflistung ist weder vollständig noch wertend.

Tab. 3: Beispiele einiger in der Schweiz erhältlicher Impfstoffe

\begin{tabular}{|c|c|c|c|}
\hline Impfung & $\begin{array}{l}\text { Zeitpunkt } \\
\text { Grundimmunisierung }\end{array}$ & Zeitpunkt Booster & Beispiele Impfstoff \\
\hline $\begin{array}{l}\text { Diphtherie/Tetanus } \\
\text { (dT) }\end{array}$ & 0,2 und 8 Monate & $\begin{array}{l}\text { Alter bis } 65 \text { Jahre: Alle } 20 \\
\text { Jahre } \\
\text { Alter ab } 65 \text { Jahre: Alle } 10 \\
\text { Jahre }\end{array}$ & $\begin{array}{l}\text { Td-Pur® }(\mathrm{dT}) \\
\text { Revaxis }{ }^{\circledR} \text { (mit IPV) }\end{array}$ \\
\hline $\begin{array}{l}\text { Pertussis } \\
\text { (pa) }\end{array}$ & 1 Injektion & $\begin{array}{l}\text { 1x im Alter zwischen } 25 \text { und } \\
29 \text { Jahren und für Personen } \\
\text { mit Kontakt zu Säuglingen } \\
<6 \text { Monaten wenn Impfung } \\
>10 \text { Jahre her }\end{array}$ & Boostrix® (mit dT) \\
\hline Masern/Mumps/Röteln & 0 und $>1$ Monat & Keine & $\begin{array}{l}\text { Priorix® } \\
\text { MMR-VaxPro® }\end{array}$ \\
\hline $\begin{array}{l}\text { Poliomyelitis } \\
\text { (IPV) }\end{array}$ & 0,2 und 8 Monate & $\begin{array}{ll}\text { Nur bei Aufenthalt in } \\
\text { Endemiegebieten alle } 10 \\
\text { Jahre }\end{array}$ & $\begin{array}{l}\text { Poliorix } \AA \\
\text { Revaxis } \AA \text { (mit dT) } \\
\text { Boostrix-Polio® (mit dTpa) }\end{array}$ \\
\hline Hepatitis B & 0,1 und 6 Monate & Keine & $\begin{array}{l}\text { Engerix® } \\
\text { HBVAXPRO® } \\
\text { Twinrix® (mit Hepatitis A) }\end{array}$ \\
\hline Influenza & Jährlich & Keine & $\begin{array}{l}\text { Inflexal® } \\
\text { Fluarix® } \\
\text { Fluad® } \\
\text { Mutagrip® }\end{array}$ \\
\hline Varizellen & 0 und $>1$ Monat & Keine & $\begin{array}{l}\text { Varivax® } \\
\text { Varilrix® }\end{array}$ \\
\hline $\begin{array}{l}\text { Humanes } \\
\text { Papillomavirus }\end{array}$ & $0,1-2$ und 6 Monate & Keine & $\begin{array}{l}\text { Gardasil® } \\
\text { Cervarix® }\end{array}$ \\
\hline FSME & $\begin{array}{l}0,1 \text { und } 6 \text { Monate bei } \\
\text { FSME-Immun® } \\
0,1 \text { und } 10 \text { Monate bei } \\
\text { Encepur® }\end{array}$ & alle 10 Jahre & $\begin{array}{l}\text { Encepur® } \\
\text { FSME-immun® }\end{array}$ \\
\hline Pneumokokken & 1 Dosis & $\begin{array}{l}\text { Ev. } 1 \text { Booster nach } 5 \text { Jahren, } \\
\text { wenn erste Impfung <65 } \\
\text { Jahre verabreicht wurde }\end{array}$ & Pneumovax® \\
\hline Hepatitis A & $\begin{array}{l}\text { Je nach Impfstoff: } \\
\text { Havrix®: } 0 \text { und } 6 \text { Monate } \\
\text { Twinrix®: } 0,1 \text { und } 6 \text { Monate }\end{array}$ & $\begin{array}{l}\text { Wahrscheinlich keine, } \\
\text { Schutz mindestens 20Jahre, } \\
\text { evtl. lebenslang }\end{array}$ & $\begin{array}{l}\text { Havrix® } \\
\text { Twinrix® (mit Hepatitis B) }\end{array}$ \\
\hline Meningokokken & 0 und 1-2 Monate & alle 5 Jahre & $\begin{array}{l}\text { Menveo® } \\
\text { Mencevac } \AA\end{array}$ \\
\hline
\end{tabular}




\section{Fallbericht zur Überprüfung des Impfschutzes beim gesunden Erwachsenen}

Q

Ein 35-jähriger Tamile stellt sich zur medizinischen Checkup-Untersuchung in einer allgemeinärztlichen Praxis in Zürich vor. Er arbeitet als Reinigungsfachkraft in einer Grossbank und lebt seit 17 Jahren in der Schweiz, Aufenthaltsbewilligung $\mathrm{C}$. Er ist verheiratet und hat einen 4 Monate alten Sohn. Mit seiner Frau zusammen pflegt er seine 70-jährige Schwiegermutter, die an einem Mammakarzinom erkrankt ist.

Vor sieben Jahren hat er sich eine Rissquetschwunde an der Stirn zugezogen, die ambulant im Spital genäht wurde. Sonst sei er immer gesund gewesen, er habe nie einen Hausarzt gehabt.
Weg zur Diagnose/Kommentar ((Ü1))

Neben der allgemein empfohlenen Beratung bezüglich Nikotin-, Alkoholkonsum und risikobehaftetem Sexualverhalten, Kontrollen von Blutdruck und Gewicht sowie altersentsprechend nötigem Screening der Lipide gehört die Impfanamnese in jede Check-Up Untersuchung! Alter, Herkunft, Beruf, persönliche Anamnese und Tätigkeiten in der Freizeit geben Hinweise auf allenfalls nötige ergänzend empfohlene Impfungen.

Weg zur Diagnose/Kommentar ((Ü1))

Das wichtigste Hilfsmittel für die Impfanamnese, der Impfausweis, ist bei diesem Patienten nicht vorhanden.

Aufgrund der Herkunft kann bei diesem jungen Mann nicht sicher davon ausgegangen werden, dass eine regelrechte Grundimmunisierung für die Basisimpfungen (diTP $\mathrm{A}_{\mathrm{a}}$, MMR und Polio) stattgefunden hat.

Aus den obigen Angaben wissen wir, dass der Patient wegen einer RQW im Spital war und können daraus schliessen, dass er höchstwahrscheinlich mindestens eine dT-Impfung erhalten hat.

Ansonsten würden wir den Mann bzgl. Polio und MMR als ungeimpft betrachten.

\section{Evaluation der Risikofaktoren ((Ü3))}

- Der Patient arbeitet als Reinigungsfachmann in einer Grossbank. In der Freizeit spielt er Cricket.
- Die Arbeit als Reinigungsfachmann ausserhalb des Gesundheitswesens birgt (ausser der Verletzungsgefahr mit Tetanusrisiko) keine spezifischen Risiken. Da der Patient sich draussen im Kanton Zürich aufhält, einem Endemiegebiet für FSME, besteht eine Indikation für FSME-Impfung. 


\begin{tabular}{|c|c|}
\hline $\begin{array}{l}\text { - Der Patient pflegt seine 70-Jährige bettlägerige } \\
\text { Schwiegermutter mit einem Mammakarzinom. }\end{array}$ & $\begin{array}{l}\text { - Bei Kontakt mit chronisch kranken Patienten mit } \\
\text { einem gewissen Grad von Immunsuppression } \\
\text { besteht die Indikation für jährliche } \\
\text { Grippeimpfungen. }\end{array}$ \\
\hline $\begin{array}{l}\text { - Der Patient verneint Risikoverhalten in der } \\
\text { Sexualanamnese, seine Ehefrau sei ebenfalls völlig gesund. }\end{array}$ & $\begin{array}{l}\text { - Die Frage nach sexuellem Risikoverhalten wird } \\
\text { gestellt, um die Indikation für eine Hepatitis-B- } \\
\text { Impfung zu prüfen. }\end{array}$ \\
\hline $\begin{array}{l}\text { - Die Frage nach durchgemachten Windpocken beantwortet } \\
\text { der Patient mit Nein. }\end{array}$ & $\begin{array}{l}\text { - Bei diesem jungen (<40-jährigen) Patienten } \\
\text { besteht die Indikation für eine Varizellen-Impfung. } \\
\text { Da die Durchseuchung mit Windpocken extrem } \\
\text { hoch ist und die Anamnese häufig falsch negativ, } \\
\text { wird vor der Impfung eine Serologie durchgeführt. }\end{array}$ \\
\hline $\begin{array}{l}\text { - Alle zwei Jahre reist der Patient nach Sri Lanka. Andere } \\
\text { Reisen werden verneint. }\end{array}$ & $\begin{array}{l}\text { - Für Reisen nach Sri Lanka ist eine Hepatitis-A- } \\
\text { Impfung empfohlen. Zur Abklärung, ob bereits eine } \\
\text { Hepatitis A durchgemacht wurde, wird eine } \\
\text { Serologie durchgeführt. }\end{array}$ \\
\hline Labor & \\
\hline Varizellen-lgG: Positiv & $\begin{array}{l}\text { - Die positive Varizellen-Serologie zeigt eine } \\
\text { durchgemachte Erkrankung, eine Impfung ist somit } \\
\text { hinfällig. }\end{array}$ \\
\hline Hepatitis A-lgG: Positiv & $\begin{array}{l}\text { - Es besteht ein Zustand nach Hepatitis } A \text {, eine } \\
\text { Impfung erübrigt sich. }\end{array}$ \\
\hline
\end{tabular}

Therapie des Patienten ((Ü1))

Basisimpfungen: Der Patient benötigt also eine Vervollständigung der Grundimmunisierung für Diphtherie und Tetanus, und eine einmalige Impfung gegen Pertussis (da er Vater eines Säuglings ist). Das Schema für «unvollständig bzw. nicht geimpfte Erwachsene» aus dem schweizerischen Impfplan kommt zur Anwendung: Diphtherie/Tetanus wurde höchstwahrscheinlich mindestens einmalig geimpft (vor sieben Jahren wegen einer $\mathrm{RQW}$ ), hier sind noch zwei weitere Impfungen nötig: Eine Dosis sofort (Boostrix® Polio) und eine Dosis (Revaxis $\AA$ ) nach sechs Monaten. Bezüglich Polio betrachten wir den Patienten als ungeimpft, es sind insgesamt drei Impfungen nötig, also (nach Boostrix ${ }^{\circledR}$ Polio und Revaxis $\AA$ ) noch eine Polioimpfung (z.B. Poliorix $®$ ) zusätzlich.

Aufgrund seines Jahrgangs qualifiziert der Patient auch zu einer Grundimmunisierung mit MMR, d.h. zwei Impfungen im Abstand von mindestens einem Monat.

Bezüglich der empfohlenen ergänzenden Impfungen aufgrund von Risikofaktoren qualifiziert er für eine Influenzaimpfung (vor der Grippesaison) und für eine FSME-Impfung.

Vor der Impfung wird der Patient über die Indikation und die möglichen Nebenwirkungen aufgeklärt. In der 
Krankengeschichte werden Aufklärung und Impfungen (Name/Lot Nummer) dokumentiert. Ein Impfbuch wird angelegt. Falls im Zusammenhang mit der Impfung Nebenwirkungen auftreten, werden diese ebenfalls dokumentiert.

Die Impfungen können am selben Tag verabreicht werden, die Injektionen sollten an verschiedenen Lokalisationen mit mindestens $2 \mathrm{~cm}$ Abstand gemacht werden. Eine Grippeimpfung wurde im folgenden November geplant. 


\section{Fragen zur Überprüfung des Impfschutzes beim gesunden Erwachsenen}

Frage 1

Wie lautet die neue Empfehlung für Auffrischimpfungen von Diphtherie/Tetanus? (Einfachauswahl, 1 richtige Antwort)

a) Booster-Impfungen für Erwachsene alle 10 Jahre für alle Altersgruppen

b) Booster-Impfungen für Erwachsene alle 20 Jahre bis zum Alter von 65 Jahren, alle 10 Jahre für über 65Jährige

c) Booster-Impfungen für Erwachsene alle 20 Jahre für alle Altersgruppen

d) Booster-Impfungen für Erwachsene alle 10 Jahre bis zum Alter von 65 Jahren, alle 20 Jahre für über 65Jährige

e) Booster-Impfungen nur bei Verletzungen

Frage 2

Welche Aussage zu Lebendimpfstoffen ist falsch? (Einfachauswahl, 1 richtige Antwort)

a) 2 Verschiedene Lebendimpfstoffe dürfen am gleichen Tag verabreicht werden.

b) Lebendimpfstoffe können mit Totimpfstoffen gleichzeitig verabreicht werden.

c) Nur bei Immunsupprimierten sollte eine Latenz von 4 Wochen zwischen 2 Lebendimpfungen eingehalten werden.

d) Lebendimpfstoffe werden in der Regel subkutan verabreicht.

e) Polio wird üblicherweise in der Schweiz mit einem Lebendimpfstoff geimpft.

\section{Frage 3}

Welche Aussage trifft zu? (Einfachauswahl, 1 richtige Antwort)
a) Tetanusimpfungen mit zu kurzem Zeitintervall zwischen den jeweiligen Impfungen führen zu zunehmenden lokalen Nebenwirkungen der Impfung.
b) Eine Pertussis-Auffrischimpfung sollte neu bei allen Altersgruppen einmalig erfolgen.
c) Schwangere dürfen gegen Varizellen geimpft werden.
d) Mitarbeiter von Hilfsorganisationen mit Tätigkeit in Schwarzafrika sollten mit BCG geimpft werden.
e) Pneumokokkenimpfungen sollten bei über 65-Jährigen alle 5-10 Jahre wiederholt werden

\section{Frage 4}

Welche Aussage trifft bzgl. Hepatitis-Impfungen zu? (Einfachauswahl, 1 richtige Antwort)
a) Eine serologische Erfolgskontrolle ist in jedem Fall nach einer Grundimmunisierung gegen Hepatitis-B angezeigt.
b) Hepatitis A sollte alle 10 Jahre aufgefrischt werden.
c) Ist vor 10 Jahren nur eine Impfung gegen Hepatitis B erfolgt, muss eine erneute Grundimmunisierung durchgeführt werden (3 Impfungen im Abstand 0,1,6 Monate).
d) Bei homosexuellen Männern übernimmt die Krankenkasse die Kosten der Hepatitis B-Impfung.
e) Hepatitis A muss nur bei Reisenden geimpft werden.

Frage 5

Für welche Patientin ist die genannte Impfindikation sicher gegeben? (Einfachauswahl, 1 richtige Antwort)

a) Varizellenimpfung bei 32-jähriger Frau mit anamnestisch durchgemachten Varizellen 
b) Masernimpfung bei 60-jähriger Schweizerin, die sich an keine durchgemachte Kinderkrankheit erinnern kann.

c) Masernimpfung bei 26-jähriger Inderin mit anamnestisch durchgemachten Masern

d) Tollwutimpfung bei 56-jähriger Züchterin von Schäferhunden.

e) Influenzaimpfung bei 38-jähriger gesunder Schalterangestellten in einer Bank.

\section{Autoren}

Dr. med. Aline Wolfensberger, Klinik und Poliklinik für Innere Medizin, Universitätsspital Zürich

Prof. Dr. med. Christoph Hatz, Abteilung für Epidemiologie und Prävention übertragbarer Krankheiten, Institut für Sozial- und Präventivmedizin, Universität Zürich und schweizerisches Tropen- und Public Health-Institut, Basel Dr. med. Maia Funk, Abteilung für Epidemiologie und Prävention übertragbarer Krankheiten, Institut für Sozialund Präventivmedizin, Universität Zürich

Dr. med. Silvana Rampini, Klinik und Poliklinik für Innere Medizin, Universitätsspital Zürich

\section{Korrespondenzadresse}

Dr. med. S. Rampini

Klinik und Poliklinik für Innere Medizin

Universitätsspital Zürich

Rämistrasse 100, 8091 Zürich

\section{((Autor: Bitte noch Email-Adresse von korresp. Autor))}

\section{Bibliographie}

1. Webpage. http://www.bag.admin.ch/ekif/04423/04428/index.html?lang=de)

2. Webpage. http://apps.who.int/immunization

3. Webpage. http://www.viavac.ch/ 
DOI 10.1024/1661-8157/a000982

((PX_15_Rubrik CME-Antworten))

\section{Antworten zu den Fragen zur Überprüfung des Impfschutzes bei gesunden Erwachsenen aus PRAXIS Nr. 14}

\section{Frage 1}

\section{Richtig ist Antwort b).}

Gemäss den neuen Richtlinien des schweizerischen Impfplans wurde für Erwachsene bis 65 Jahre das Intervall für die Booster-Impfungen auf 20 Jahre verlängert. Dies mindert die Gefahr der Hyperimmunisierung und der Impfschutz ist trotzdem gegeben. Für ältere Personen über 65 Jahre ist das Intervall weiterhin zehn Jahre.

\section{Frage 2}

\section{Richtig ist Antwort e).}

Poliomyelitis wird nicht mehr mit einem Lebendimpfstoff, sondern nur noch mit einem inaktivierten Impfstoff (IPV) geimpft. So konnten die seltene, aufgrund der oralen Impfung mit attenuierten Viren auftretende Impfpolio eliminiert werden.

Ad a) bis d): Lebendimpfstoffe können bei gesunden Erwachsenen sowohl untereinander kombiniert, als auch kombiniert mit Totimpfstoffen verabreicht werden. Nur bei Immunsupprimierten sollten Lebendimpfstoffe nicht gleichzeitig, sondern in einem Abstand von mindestens vier Wochen gegeben werden. Die Impfung erfolgt subkutan.

\section{Frage 3}

\section{Richtig ist Antwort a).}

Aufgrund der Hyperimmunisierung kommt es bei zu häufig verabreichten Tetanus-Booster-Impfungen zu verstärkter Lokalreaktion wie Schwellung, Rötung und Dolenz.

Ad b): Falsch, da eine Pertussis-Auffrischimpfung zwar neu auch bei Erwachsenen durchgeführt werden soll, aber nur bei zwischen 25- und 29-Jährigen sowie bei Personen, die mit Säuglingen unter sechs Monaten Kontakt haben.

Ad c): Falsch, da die Varizellenimpfung zu den Lebendimpfstoffen gehört und bei Schwangeren somit nicht verabreicht werden darf.

Ad d): Falsch, es gibt keine Indikation für BCG-Impfungen für Erwachsene.

Ad e): Falsch, da bei der Immunisierung gegen Pneumokokken eine zweite Impfung nur erfolgen sollte, wenn die erste Impfung im Alter unter 65 Jahre stattgefunden hat und länger als fünf Jahre her ist.

\section{Frage 4}

\section{Richtig ist Antwort d).}

Die Hepatitis-B-Impfung wird bei Patienten mit gegebener Risikosituation (so bei homosexuellen Männern) von der Krankenkasse bezahlt.

Ad a) und c): Eine Impferfolgskontrolle für Hepatitis B braucht es nur bei Angestellten im Gesundheitswesen oder bei Verdacht auf mangelnde Immunisierung, nicht grundsätzlich. Eine begonnene Grundimmunisierung muss nie bei Null begonnen werden, da jede Impfung zählt!

Ad b) und e): Es gibt neben Auslandreisen auch andere Indikationen für eine Hepatitis-A-Impfung, z.B. eine Tätigkeit als Kanalisationsarbeiter, Personen mit chronischen Lebererkrankungen etc. Die Dauer des 
Impfschutzes für Hepatitis A ist noch unklar. Man geht davon aus, dass er mindestens für 20 bis 30 Jahre gegeben ist.

\section{Frage 5}

\section{Richtig ist Antwort c).}

Anamnestisch durchgemachte Masern oder Röteln entbinden bei Patienten mit Jahrgang 1964 oder jünger nicht von einer Impfung! Die Patientin aus Antwort c) muss also gegen MMR geimpft werden. Hingegen kann auch bei negativer Krankheitsanamnese bei Patienten mit Jahrgang 1963 oder älter von einer Immunität bzgl. Masern, Mumps und Röteln ausgegangen werden. Die einzige Kinderkrankheit mit verlässlicher Anamnese sind die Varizellen, Antwort a) ist somit falsch. Die Hunde-Züchterin muss nicht gegen Tollwut geimpft werden, eine Tollwutimpfung ist nur für Tierärzte und andere Risikoberufe, sowie für Auslandreisende mit speziellem Risiko indiziert. Die Indikationen für Influenza-Impfungen sind im Impfplan aufgelistet, gesunde Schalterangestellte gehören nicht zu der Indikationsgruppe. 